\title{
Quasi-Static Folding and Deployment of Ultrathin Composite Tape-Spring Hinges
}

\author{
H. M. Y. C. Mallikarachchi* \\ University of Cambridge, Cambridge, England CB2 1PZ, United Kingdom \\ and \\ S. Pellegrino \\ California Institute of Technology, Pasadena, California 91125 \\ DOI: $\underline{10.2514 / 1.47321}$
}

\begin{abstract}
Deployable structures made from ultrathin composite materials can be folded elastically and are able to selfdeploy by releasing the stored strain energy. This paper presents a detailed study of the folding and deployment of a tape-spring hinge made from a two-ply plain-weave laminate of carbon-fiber reinforced plastic. A particular version of this hinge was constructed, and its moment-rotation profile during quasi-static deployment was measured. The present study is the first to incorporate in the simulation an experimentally validated elastic micromechanical model and to provide quantitative comparisons between the simulations and the measured behavior of an actual hinge. Folding and deployment simulations of the tape-spring hinge were carried out with the commercial finite element package Abaqus/Explicit, starting from the as-built unstrained structure. The folding simulation includes the effects of pinching the hinge in the middle to reduce the peak moment required to fold it. The deployment simulation fully captures both the steady-state moment part of the deployment and the final snap back to the deployed configuration. An alternative simulation without pinching the hinge provides an estimate of the maximum moment that could be carried by the hinge during operation. This is about double the snapback moment.
\end{abstract}

\begin{tabular}{|c|c|}
\hline \multicolumn{2}{|r|}{ Nomenclature } \\
\hline$a$ & $=$ maximum tow thickness, $\mathrm{mm}$ \\
\hline$A B D, A B D^{\prime}$ & $\begin{array}{l}=\text { constitutive matrices in coordinate system } x \text { and } \\
y \text { and } x^{\prime} \text { and } y^{\prime}\end{array}$ \\
\hline$A_{i j}$ & $\begin{aligned}= & \text { coefficients of upper left } 3 \times 3 \text { submatrix of } \\
& A B D, \mathrm{~N} / \mathrm{mm}\end{aligned}$ \\
\hline$a_{i j}$ & $\begin{array}{l}=\text { coefficients of in-plane compliance matrix, } \\
\mathrm{mm} / \mathrm{N}\end{array}$ \\
\hline$B_{i j}$ & $\begin{aligned}= & \text { coefficients of upper right } 3 \times 3 \text { submatrix of } \\
& A B D, \mathrm{~N}\end{aligned}$ \\
\hline$c_{d}$ & $=$ dilatational wave speed, $\mathrm{mm} / \mathrm{s}$ \\
\hline$c_{v}$ & $=$ viscous pressure coefficient, $\mathrm{Ns} / \mathrm{mm}^{3}$ \\
\hline$D_{i j}$ & $\begin{aligned}= & \text { coefficients of lower right } 3 \times 3 \text { submatrix of } \\
& A B D, \mathrm{~N} \cdot \mathrm{mm}\end{aligned}$ \\
\hline$E_{b}$ & $=$ energy balance, $\mathrm{mJ}$ \\
\hline$E_{i}$ & $=$ internal energy, $\mathrm{mJ}$ \\
\hline$E_{k}$ & $=$ kinetic energy, $\mathrm{mJ}$ \\
\hline$E_{m}^{n}$ & $=$ elastic modulus of matrix, $\mathrm{N} / \mathrm{mm}^{2}$ \\
\hline$E_{v}$ & $=$ viscous dissipation, $\mathrm{mJ}$ \\
\hline$E_{w}$ & $=$ work done by external forces, $\mathrm{mJ}$ \\
\hline$E_{1}$ & $=$ longitudinal stiffness of tow, $\mathrm{N} / \mathrm{mm}^{2}$ \\
\hline$E_{2}, E_{3}$ & $=$ transverse stiffnesses of tow, N/mm $\mathrm{mm}^{2}$ \\
\hline$G_{12}, G_{13}$ & $=$ shear stiffnesses of tow, $\mathrm{N} / \mathrm{mm}^{2}$ \\
\hline$G_{23}$ & $=$ in-plane shear stiffness of tow, $\mathrm{N} / \mathrm{mm}^{2}$ \\
\hline$h$ & $=$ thickness of shell elements, $\mathrm{mm}$ \\
\hline
\end{tabular}

Presented as Paper 2008-2053 at the 49th AIAA/ASME/ASCE/AHS/ASC Structures, Structural Dynamics, and Materials Conference, Schaumburg, IL, 7-10 April 2008; received 22 September 2009; revision received 30 June 2010; accepted for publication 16 July 2010 . Copyright $\odot 2010$ by H. M. Y. C. Mallikarachchi and S. Pellegrino. Published by the American Institute of Aeronautics and Astronautics, Inc., with permission. Copies of this paper may be made for personal or internal use, on condition that the copier pay the $\$ 10.00$ per-copy fee to the Copyright Clearance Center, Inc., 222 Rosewood Drive, Danvers, MA 01923; include the code 0022-4650/11 and $\$ 10.00$ in correspondence with the CCC.

${ }^{*}$ Research Student, Department of Engineering, Trumpington Street; currently Visiting Student, California Institute of Technology, Pasadena, CA 91125 .

${ }_{\dagger}^{\dagger}$ Joyce and Kent Kresa Professor of Aeronautics and Professor of Civil Engineering, Graduate Aerospace Laboratories, 1200 East California Boulevard, Mail Code 301-46; sergiop@ caltech.edu. Fellow AIAA. $l$

$l_{\min }$

$m$

$N$

n

$p$

$p_{b}$

U

$u, v, w$

$\boldsymbol{v}$

$v_{f}$

$X, Y, Z$

$x, y, z$

$x^{\prime}, y^{\prime}$

$\alpha$

$\beta$

$\gamma_{x y}$

$\Delta L$

$\varepsilon$

$\dot{\epsilon}_{v}$

$\theta$

$\kappa$

$\dot{\kappa}$

$\kappa_{x y}$

$v_{m}$

$v_{12}, v_{13}, v_{23}$

$\xi$

$\rho$

$\phi$

$(\ldots)_{x}, \ldots$

$(\ldots)^{x}, \ldots$

$(\ldots)^{\prime}$
$=$

$=$

$=$

$=$ $\mathrm{N} / \mathrm{mm}$

$=$ force per unit length stress resultant, $\mathrm{N} / \mathrm{mm}$

$=$ unit surface normal

$=$ viscous pressure, $\mathrm{N} / \mathrm{mm}^{2}$

$=$ bulk viscosity pressure, $\mathrm{N} / \mathrm{mm}^{2}$

$=$ global translation components of tape-spring hinge model, $\mathrm{mm}$

$=$ translations in $x, y$, and $z$ directions, $\mathrm{mm}$

$=$ velocity vector, $\mathrm{mm} / \mathrm{s}$

$=$ overall fiber volume fraction

$=$ global coordinate system of tape-spring hinge model

$=$ coordinate system for unit cell

$=$ curvilinear coordinate system aligned with longitudinal and circumferential directions of tape-spring hinge

$=$ time scaling factor

$=$ mass scaling factor

$=$ engineering shear strain

$=$ length of unit cell, $\mathrm{mm}$

$=$ normal midplane strain

$=$ volumetric strain rate, $1 / \mathrm{s}$

$=$ rotation, $\mathrm{rad}$

= curvature, $1 / \mathrm{mm}$

$=$ twice the mean curvature rate, $1 / \mathrm{mm} \cdot \mathrm{s}$

$=$ twice the surface twist, $1 / \mathrm{mm}$

$=$ Poisson's ratio of matrix

$=$ Poisson's ratios of tow

$=$ fraction of critical damping in highest frequency mode

$=$ density, $\mathrm{kg} / \mathrm{mm}^{3}$

$=$ angle between tow directions $(x$ and $y)$ and curvilinear coordinate system $\left(x^{\prime}\right.$ and $\left.y^{\prime}\right)$, deg

$=$ components in direction $x$, etc.

$=$ deformation directions for unit cell

$=$ quantity that refers to coordinate systems $x^{\prime}, y^{\prime}$, and $z^{\prime}$ 


\section{Introduction}

D EPLOYABLE structures made from ultrathin composite materials can be folded elastically and are able to self-deploy by releasing the stored strain energy. They are becoming more widespread because of their lower mass-to-deployed-stiffness ratio, good packaging properties, and lower cost due to a smaller number of component parts and ease of manufacture [1]. Examples of structures of this kind that have been already flown include the Boeing springback reflectors on the mobile satellite system (MSAT) $[2,3]$, the Tracking and Data Relay Satellites H, I, and J, $\underline{\underline{s}}$ and the three Northrop Grumman Astro Aerospace flattenable foldable tubes forming the Mars Advanced Radar for Subsurface and Ionosphere Sounding (MARSIS) antenna on the Mars Express spacecraft [4]. Novel solar array and reflector antenna concepts based on the same general approach have been proposed, including the hollow solid reflector structure [5] shown in Fig. 1, the fold integrated thin-film stiffener solar array, which undergoes three different folding stages to achieve a highly compacted configuration [6], and the folding large antenna tape-spring radar concept [7].

The deployment behavior of all of these structures may appear to be simple, as it relies on the natural tendency of any elastically folded structure to spring open and reach toward a configuration with zero strain energy, but in reality, these structures have to be designed with great care and attention to detail in order to produce designs that will avoid any permanent deformation or fracture of the material during folding, stowage, and deployment. Dynamic effects associated with deployment and self-latching of the structure, viscous relaxation during stowage, temperature effects during stowage and deployment, etc. also need to be considered in the design process, but they will not be considered in the present study.

This paper presents a detailed study of a foldable structure that poses many of the general challenges common to ultrathin composite stored-energy deployable structures. The particular example that is chosen consists of a thin-walled tube made of carbon-fiber reinforced plastic with an elastically foldable central section made by cutting two longitudinal slots with round ends, as shown in Fig. 2. A variant of this hinge design, with three slots, was analyzed with the implicit finite element code Abaqus/Standard, by Yee and Pellegrino [8] and Soykasap [9]. Also, each folding section of the MARSIS booms [10] is, in fact, a tape-spring hinge with two slots with enlarged round ends. Here, we present a study of a particular hinge design for which we have carried out both quasi-static deployment tests using a bending rig as well as folding and deployment simulations with the commercial finite element package Abaqus/Explicit [11]. The present study is the first to incorporate into the simulation an experimentally validated elastic micromechanical model of the woven fiber composite material and to provide quantitative comparisons between the simulations and the measured behavior of an actual hinge.

The paper is arranged as follows. Section II describes the construction of a tape-spring hinge and presents a quasi-static deployment experiment that was carried out. Section III presents the experimentally validated linear-elastic material model that was adopted for the woven composite laminate of the tape-spring hinge. This model takes the form of a $6 \times 6$ stiffness matrix that is derived from homogenization theory. The finite element model of the repeating unit cell of the laminate is presented, and the technique for imposing periodic boundary conditions is summarized. Sections IV and V present the finite element model of the hinge and the simulation techniques to carry out quasi-static folding and deployment simulations with Abaqus/Explicit. Section VI describes the simulation of a tape-spring hinge. Section VII presents a detailed study of the sensitivity of various results of the simulation to a set of adjustable parameters; the outcome of this study is a robust procedure for running Abaqus/Explicit dynamic analyses that provide an accurate representation of quasi-static folding and deployment

Data available at http://www.boeing.com/defense-space/space/bss/ factsheets $/ 601 / \mathrm{msat} / \mathrm{msat} . \mathrm{html}$ [retrieved 12 November 2010].

${ }^{\S}$ Data available at http://www.boeing.com/defense-space/space/bss/ factsheets/601/tdrs_hij/tdrs_hij.html [retrieved 12 November 2010]. behavior. Section VIII presents an analysis of the midsurface strain and curvature distribution in any configuration of the deformed structure. Based on these results, an analysis of the unit cell of the laminate provides the maximum strains in the fibers and the matrix; estimates of the margin of safety against failure can be determined by comparing these peak values to the material failure values. Section IX considers the behavior of a tape-spring hinge that is subject only to end rotations. A folding and deployment cycle for this loading condition provides an estimate of the peak moment that could be applied to the deployed hinge (if material failure does not occur first) and captures the snap-through and reverse snap behavior of the hinge. Section $X$ compares deployment simulation and experimental results for the hinge; it also includes a comparison of the predicted and actual measured moment-rotation profiles during deployment. Section XI concludes the paper.

\section{Deployment Experiment}

A quasi-static deployment experiment was carried out on a tapespring hinge with an internal diameter of $38 \mathrm{~mm}$ and a thickness of $0.2 \mathrm{~mm}$. The tape-spring hinge wall had an areal density of $260 \mathrm{~g} / \mathrm{m}^{2}$. It consisted of a two-ply \pm 45 plain-weave laminate of $1 \mathrm{k}$ tows of T300 carbon fibers impregnated with HexPly 913 epoxy resin. The tape-spring hinge was manufactured by laying two plies of resin-impregnated fabric on a $38-\mathrm{mm}$-diam steel mandrel sprayed with polytetrafluoroethylene (PTFE) release agent. The impregnated fabric was wrapped in PTFE release film, placed inside a heatshrinking sleeve, and cured under vacuum for $1 \mathrm{~h}$ at $125^{\circ} \mathrm{C}$ and $600 \mathrm{kPa}$. After cooling, the mandrel was pulled out of the tube, and two parallel slots were cut in the tube with a pressurized water jet machine. The geometry of the folding section of the tape-spring hinge consisted of two 140-mm-long 25-mm-wide tape springs separated by 110 -mm-long (from center to center) 30-mm-wide slots with semicircular ends.

Folding of this tape-spring hinge is best initiated by pinching it in the middle to avoid high bending moments that may damage the hinge. Once the height of the central region has been approximately halved, the ends of the tape-spring hinge can be rotated in opposite directions.

Deployment tests were carried out by attaching a pair of tube holders to the apparatus, previously used by Seffen and Pellegrino, to study the behavior of tape springs [12]. This apparatus consists of two small gear boxes with a reduction ratio of 80 , one attached to a rigid base and the other mounted on a linear bearing, supporting hollow strain-gauged shafts. The base rotation of the shafts is measured with a resolution of $0.045 \mathrm{deg}$ and to an accuracy of $\pm 0.4 \mathrm{deg}$ (due to backlash). The ends of the tape-spring hinge are attached to the shafts, which behave as load cells with a linear response up to $1.10 \mathrm{Nm}$ and an accuracy of $\pm 2 \mathrm{Nmm}$.

Before starting the test, the strain gauge readings were set to zero in the deployed (i.e., unstressed) configuration, shown in Fig. 3. Then, the tape-spring hinge was pinched in the middle and folded by rotating the ends by equal amounts. During the test, the ends were rotated back in small steps while keeping the end moments roughly equal. This was done by rotating the moving gear box by the desired amount and then rotating the fixed gear box to equalize the end moments.

At the end of the test, the moment-rotation profile was obtained by plotting the folding angle $\theta$, defined as the difference between the end rotations and the corresponding moment at each step (Fig. 4). It can be seen that the bending moment is approximately constant until the final snapback, apart from a small hump for $\theta \approx 40$ deg caused by the loss of contact between the internal and external tape springs, in the fold region. Note that the particular tape-spring hinge design that was tested had a unique equilibrium configuration when the deployment moment was zero.

\section{Model of Laminate}

Thin laminates made from woven tows of fibers are not modeled accurately by classical laminate theory [13]. Hence, the material of 


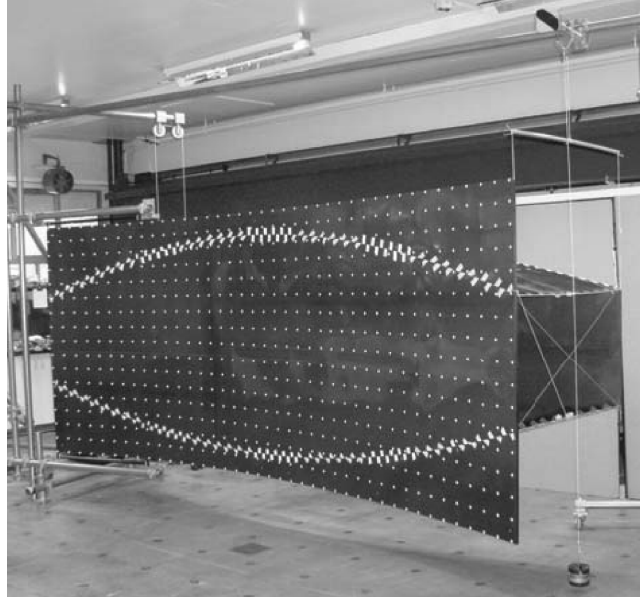

a)

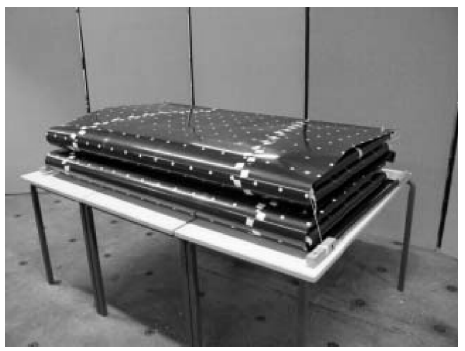

b)

Fig. 1 Hollow solid reflector: a) deployed and b) folded.

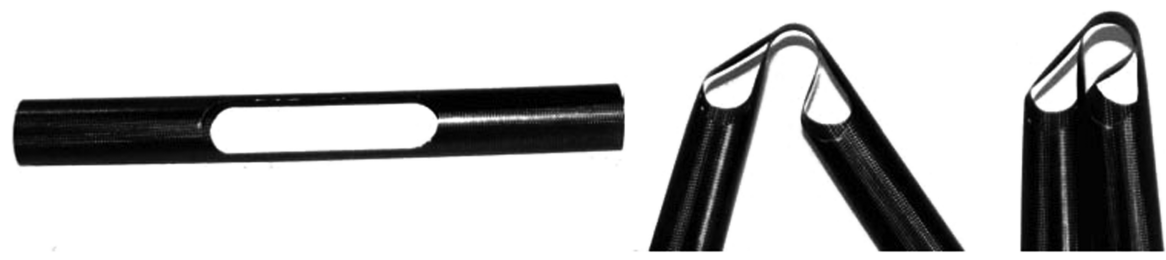

Fig. 2 Photos of tape-spring hinge deployed, partially folded, and fully folded.

the tape-spring hinge has been modeled as a linear-elastic thin Kirchhoff plate for which the properties are defined by a homogenization technique.

The geometric properties of the two-ply laminate were calculated from micrographs of several cross sections, and the overall fiber volume fraction $v_{f}=0.68$ was calculated from the weight of cured composite material and resin. The particular composite used for the tape-spring hinge, described in Sec. II, has a weave length of $\Delta L=2.664 \mathrm{~mm}$, a tow cross-sectional area of $0.0522 \mathrm{~mm}^{2}$, and a maximum tow thickness of $a=0.059 \mathrm{~mm}$. The mechanical properties of the tows and resin are defined in Table 1 .

In the simplest micromechanics model, the tows were modeled as wavy beams having an equivalent rectangular cross-sectional area [14], but in this model, the beams are constrained only along their centerlines, and so the distributed nature of the constraint provided across the tow width is neglected. Hence, this model did not capture Poisson's effects accurately; in particular, it provided rather poor predictions of $A_{12}$ and gave $D_{12}=0$. Then, several solid models were investigated by extending the work on single-ply plain-weave laminates by Karkkainen and Sankar [15]. The model that was finally chosen has the two plies in phase and consists of six-node triangular prisms; it is shown in Fig. $\underline{5}$. Note that the $x, y$, and $z$ coordinate

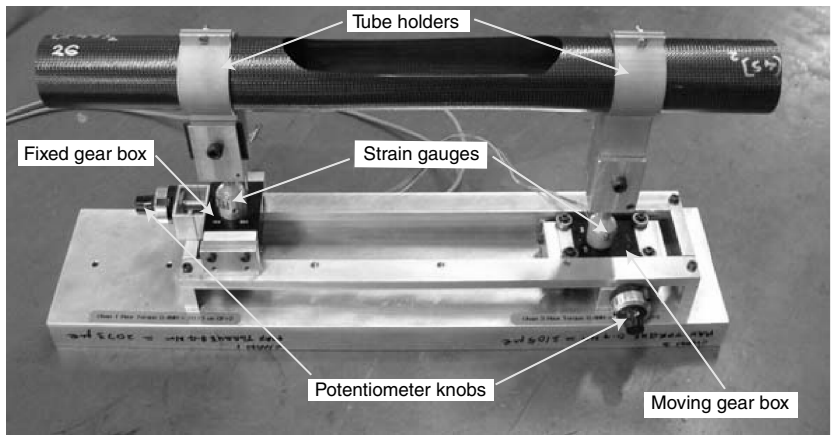

Fig. 3 Quasi-static deployment test rig. system is such that the tow centerlines lie in planes parallel to $x z$ and $y z$. The surfaces of each tow are defined by

$$
z=a\left( \pm 2 \pm \sqrt[4]{\left|\sin \left(\frac{2 \pi x}{\Delta L}\right)\right|} \pm \sqrt[4]{\left|\sin \left(\frac{2 \pi y}{\Delta L}\right)\right|}\right)
$$

where the sign of the first term is + for the upper ply and - for the lower ply; the signs of the second and third terms are $(+,+)$ for the top surface of the ply, $(+,-)$ or $(-,+)$ for the interface between the two tows, and $(-,-)$ for the lower surface of the ply.

This representation of the tow surfaces ensures a perfect geometric match between tows in the same ply; however, tows in different plies touch only at four points, and hence there is a gap that has been filled with six-node triangular prisms modeling additional neat resin. The fiber volume fraction of the tows was left unchanged, and so the resulting overall volume fraction was $v_{f}=0.62$.

The particular model shown in Fig. $\underline{5}$ consists of 1520 and 3200 six-node triangular prisms elements, respectively, for each tow and for the additional resin. The boundary nodes were attached by means of rigid vertical beams to dummy nodes lying in the midplane. Each

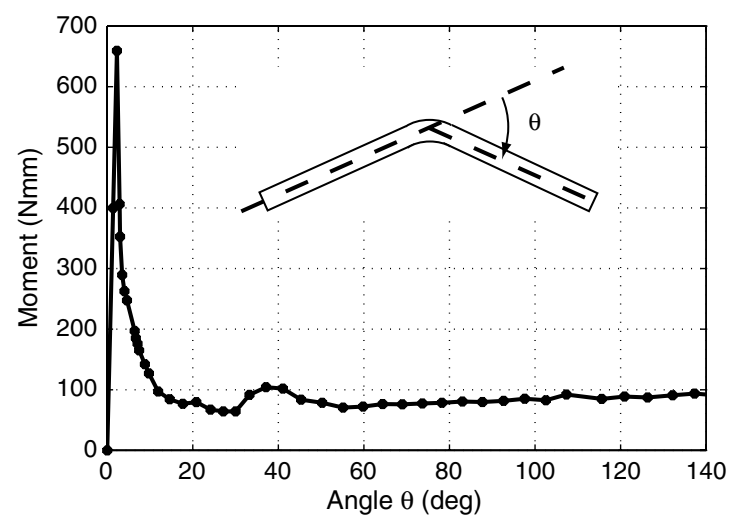

Fig. 4 Moment-rotation profile measured during deployment. 
Table 1 Properties of cured T300-1k/913 tow and HexPly 913 resin

\begin{tabular}{lcc}
\hline \hline Properties & Value \\
\hline & Tow & \\
$E_{1}, \mathrm{~N} / \mathrm{mm}^{2}$ & & 159,520 \\
$E_{2}=E_{3}, \mathrm{~N} / \mathrm{mm}^{2}$ & 11,660 \\
$G_{12}=G_{13}, \mathrm{~N} / \mathrm{mm}^{2}$ & 3813 \\
$G_{23}, \mathrm{~N} / \mathrm{mm}^{2}$ & 3961 \\
$\nu_{12}=v_{13}$ & 0.27 \\
$\nu_{23}$ & 0.47 \\
$\rho, \mathrm{kg} / \mathrm{mm}^{3}$ & & $1.6 \times 10^{-6}$ \\
$E_{m}, \mathrm{~N} / \mathrm{mm}^{2}$ & Resin & 3390 \\
$\nu_{m}$ & & 0.41 \\
\hline \hline
\end{tabular}

dummy node was related to the corresponding dummy node opposite by means of a constraint equation that enforced periodic boundary conditions. These equations prescribed the relative displacements and rotations of the dummy nodes in terms of the midplane strains and out-of-plane curvatures:

$$
\begin{gathered}
\varepsilon_{x}=\frac{\partial u}{\partial x} \\
\varepsilon_{y}=\frac{\partial v}{\partial y} \\
\gamma_{x y}=\frac{\partial u}{\partial y}+\frac{\partial v}{\partial x} \\
\kappa_{x}=-\frac{\partial^{2} w}{\partial x^{2}} \\
\kappa_{y}=-\frac{\partial^{2} w}{\partial y^{2}} \\
\kappa_{x y}=-2 \frac{\partial^{2} w}{\partial x \partial y}
\end{gathered}
$$

of the homogenized Kirchhoff plate. It should be noted that the engineering shear strain and twice the surface twist have been used in the definition of these deformation variables.

The constraint equations are as follows:

$$
\Delta u^{x}=\varepsilon_{x} \Delta L
$$

$$
\Delta v^{x}=\frac{1}{2} \gamma_{x y} \Delta L
$$

$$
\Delta u^{y}=\frac{1}{2} \gamma_{x y} \Delta L
$$

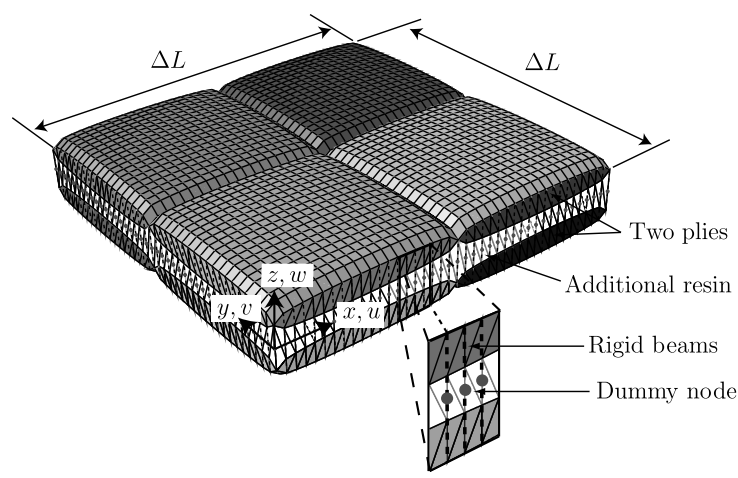

Fig. 5 Finite element model for unit cell of two-ply laminate (with $3 X$ magnification in the $z$ direction).

$$
\Delta v^{y}=\varepsilon_{y} \Delta L
$$

$$
\Delta w^{x}=-\frac{1}{2} \kappa_{x y} y \Delta L
$$

$\Delta w^{y}=-\frac{1}{2} \kappa_{x y} x \Delta L$

$\Delta \theta_{x}^{x}=-\frac{1}{2} \kappa_{x y} \Delta L$

$$
\Delta \theta_{y}^{x}=\kappa_{x} \Delta L
$$

$\Delta \theta_{x}^{y}=-\kappa_{y} \Delta L$

$$
\Delta \theta_{y}^{y}=\frac{1}{2} \kappa_{x y} \Delta L
$$

$$
\Delta \theta_{x}^{z}=0
$$

$$
\Delta \theta_{y}^{z}=0
$$

where subscripts denote the deformation directions and superscripts denote the direction of a pair of boundary nodes with equal $x$ or $y$ coordinates.

The constitutive relationship for the homogenized plate, in the coordinate directions defined in Fig. $\underline{5}$, is written in the form

$$
\left\{\begin{array}{c}
N_{x} \\
N_{y} \\
N_{x y} \\
-- \\
M_{x} \\
M_{y} \\
M_{x y}
\end{array}\right\}=\left(\begin{array}{lllllll}
A_{11} & A_{12} & A_{16} & \mid & B_{11} & B_{12} & B_{16} \\
A_{21} & A_{22} & A_{26} & & B_{21} & B_{22} & B_{26} \\
A_{61} & A_{62} & A_{66} & \mid & B_{61} & B_{62} & B_{66} \\
-- & -- & -- & -- & -- & -- & -- \\
B_{11} & B_{21} & B_{61} & \mid & D_{11} & D_{12} & D_{16} \\
B_{12} & B_{22} & B_{62} & \mid & D_{21} & D_{22} & D_{26} \\
B_{16} & B_{26} & B_{66} & \mid & D_{61} & D_{62} & D_{66}
\end{array}\right)\left\{\begin{array}{c}
\varepsilon_{x} \\
\varepsilon_{y} \\
\gamma_{x y} \\
-- \\
\kappa_{x} \\
\kappa_{y} \\
\kappa_{x y}
\end{array}\right\}
$$

The $6 \times 6$ constitutive matrix is denoted by $A B D$, and its $3 \times 3$ submatrices are denoted by $A, B$, and $D$.

The entries of the $A B D$ matrix for the two-ply laminate were computed using Virtual Work [15,16], after carrying out six separate Abaqus/Standard analyses, each corresponding to a unit amplitude of the six deformation variables, $\varepsilon_{x}, \ldots, \kappa_{x y}$. The following $A B D$ matrix was thus obtained:

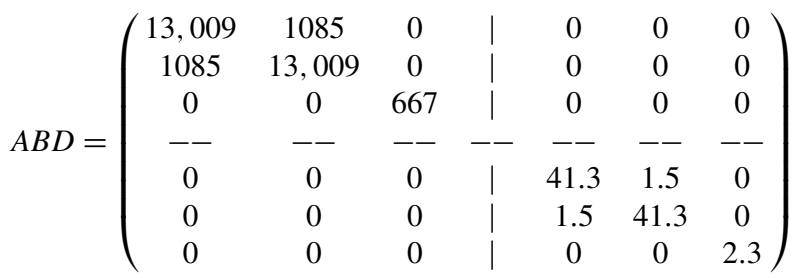

where the units are newtons and millimeters.

The constitutive matrix $A B D^{\prime}$ in the longitudinal and circumferential directions of the tape-spring hinge, at $45 \mathrm{deg}$ to $x$ and $y$, is obtained by a +45 deg rotation of the $A B D$ matrix in Eq. (5), which gives

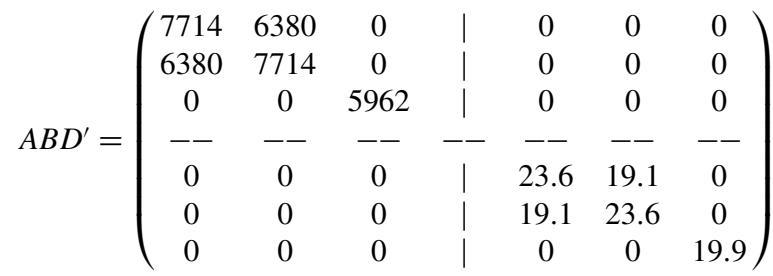


The constitutive matrices in Eqs. (ㅁ) and (ㅁ) were verified by carrying out tension tests and four-point bending tests on both $[0 / 90]_{2}$ and $[ \pm 45]_{2}$ laminates. The tension tests were done on 100mm-long and 20-mm-wide flat specimens; the four-point bending tests were carried out on 100-mm-long and 50-mm-wide specimens. Both sets of tests were done on an Instron 5569 materials testing machine with $1 \mathrm{kN}$ and $10 \mathrm{~N}$ load cells, respectively, for the tension and bending tests. All measurements of strain and deflection were made with Epsilon LE01 and LE05 laser extensometers.

Figures $6 \mathrm{a}$ and $6 \mathrm{~b}$ characterize the axial stiffness and the Poisson's ratio of three $\left[ \pm 4 \overline{5]_{2}}\right.$ laminate specimens. Figure $6 \mathrm{c}$ characterizes the bending stiffness. Each response is approximately linear, and the average behavior is shown by a black dashed line. The bending tests were done on two specimens only, but the tests were repeated after flipping each specimen upside down.

The use of narrow specimens in the tension tests ensures that the transverse and shear stress resultants are negligibly small (i.e., $N_{y^{\prime}} \approx N_{x^{\prime} y^{\prime}} \approx 0$ ) in the middle of the specimen. Hence, denoting by $a_{i j}$ the entries of the in-plane compliance matrix obtained by inverting the upper left $3 \times 3$ submatrix of $A B D^{\prime}$, one obtains

$$
\varepsilon_{x^{\prime}}=a_{11} N_{x^{\prime}}
$$

Therefore, $1 / a_{11}$ can be compared with the measured ratio $N_{x^{\prime}} / \varepsilon_{x^{\prime}}$.

In the four-point bending tests, wider specimens were used; hence, the transverse and twisting curvatures were negligibly small (i.e., $\kappa_{y^{\prime}} \approx \kappa_{x^{\prime} y^{\prime}} \approx 0$ ), and so the measured ratio $M_{x^{\prime}} / \kappa_{x^{\prime}}$ can be directly compared with $D_{11}^{\prime}$ in Eq. (ㅁ) .

A comparison of the experimental results to the predictions based on Eq. (6) is presented in Table 2, where it can be seen that there is almost perfect agreement.

\section{Tape-Spring Hinge Finite Element Model}

A finite element model of the tape-spring hinge was set up in the Abaqus/Explicit finite element package (see Fig. 7). To simulate the deployment behavior observed in Sec. II, one needs to first compute the folded configuration of the hinge, and this requires that two cross sections of the tape-spring hinge be rotated in opposite directions until the fully folded configuration is reached. It is important to note that the boundary conditions imposed at these cross sections may significantly affect the results of the simulations. Defining two rigid rings and rotating them would prevent the cross sections from deforming when the tape-spring hinge is folded. To avoid this effect, the actual stiffness of the holders used in the experiment was incorporated in the model, and the rotations were imposed through these elastic holders.

The tape-spring hinge model consisted of 2546 nodes and 2412 shell elements (S4) with a minimum element length of around $3 \mathrm{~mm}$. The model of each holder consisted of 182 nodes and 156 S4 shell elements with approximate element lengths of $5 \mathrm{~mm}$. The nodes of the holder models were rigidly attached to the nodes of the tapespring hinge model using tie constraints.

The elastic properties of the shell elements were defined in a cylindrical coordinate system (with directions $x^{\prime}, y^{\prime}$, and $z$, respectively, longitudinal, circumferential, and radial) by assigning the $A B D^{\prime}$ matrix of Eq. (6) with the shell general section parameter.

To simulate the equal end moment conditions imposed during the deployment experiment, two reference nodes $\mathrm{A}$ and $\mathrm{B}$ were attached to a dummy node $\mathrm{C}$ using the Abaqus command equation, and these reference nodes were rigidly attached to the nearest eight nodes of the holders. The sensitivity studies in Sec. VII were done with the reference nodes attached to a single node.

The boundary conditions (see Fig. 7) were defined as follows: all degrees of freedom at node A were restrained, except the rotation about the global $X$ axis. Node B was only allowed to translate along the global $Z$ axis and rotate about the $X$ axis. To keep the end moments equal, the rotational degrees of freedom of nodes A and B were restrained by prescribing the condition

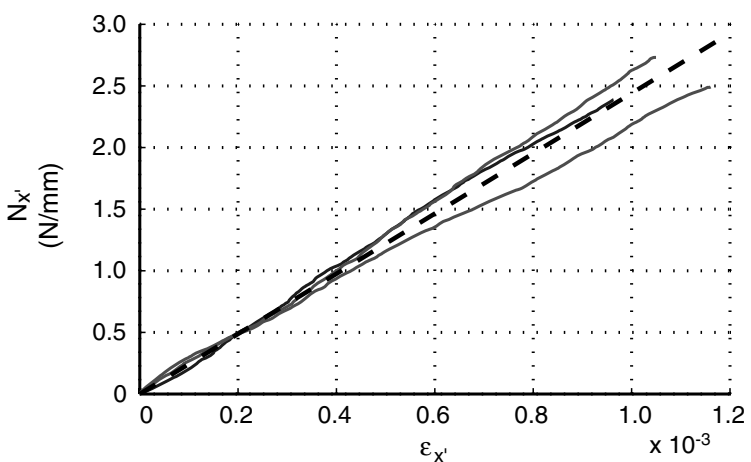

a) Tensile stiffness

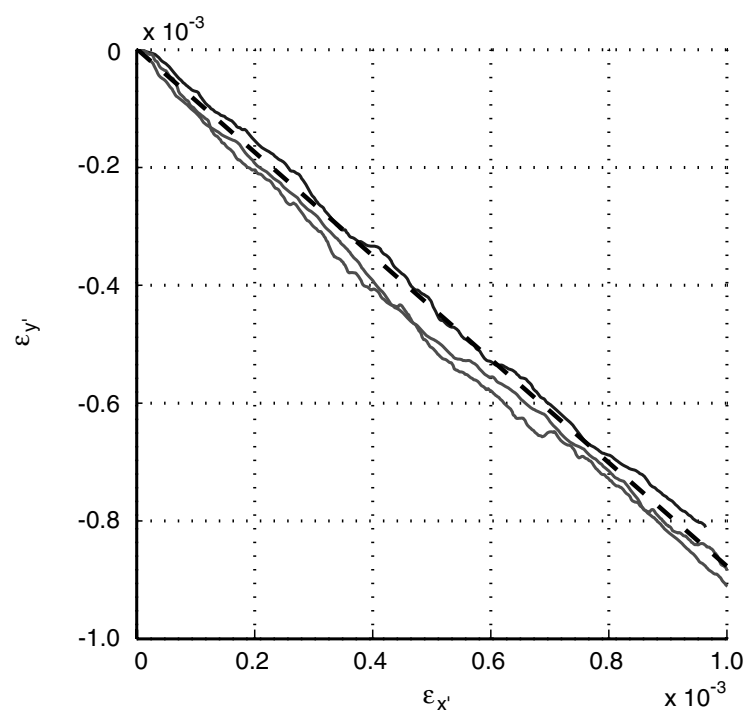

b) Poisson's ratio

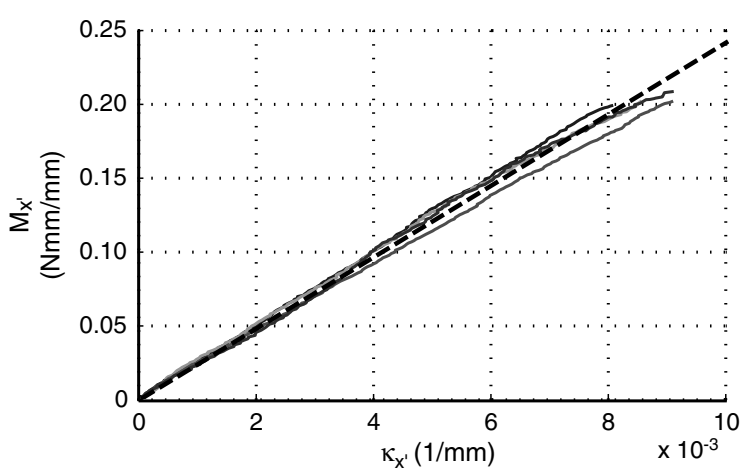

c) Bending stiffness

Fig. 6 Stiffness measurements on $[ \pm 45]_{2}$ laminates.

$$
\theta_{X}^{A}-\theta_{X}^{B}=\theta_{X}^{C}
$$

where $\theta_{X}$ denotes a rotation about the global $X$ axis.

To simulate the folding process, the folding angle $\theta_{X}^{C}$ was incremented from 0 to $170 \mathrm{deg}$ (or in some cases, only $160 \mathrm{deg}$ ) over a suitably chosen time interval; details are provided in Sec. VI.

Table 2 Comparison of predicted and measured stiffnesses of $[ \pm 45]_{2}$ laminate

\begin{tabular}{lcc}
\hline \hline & Equation (6) & Experiment \\
\hline Axial stiffness, N/mm & 2437 & 2440 \\
Poisson's ratio & 0.83 & 0.88 \\
Bending stiffness, Nmm & 23.6 & 24.2 \\
\hline \hline
\end{tabular}




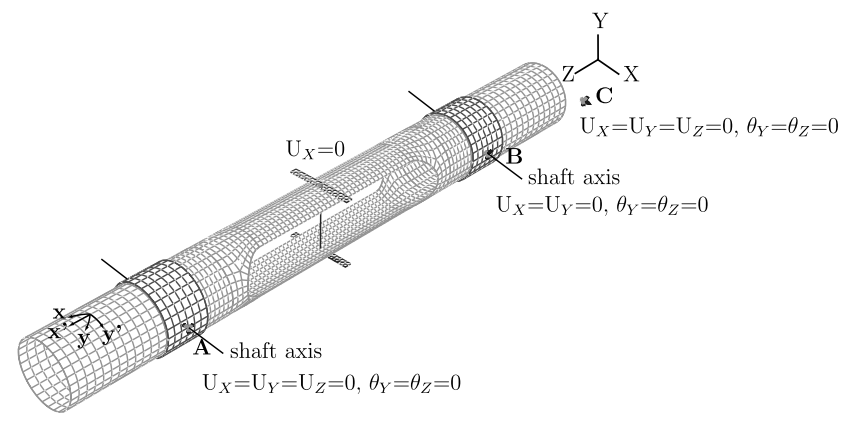

Fig. 7 Finite element model of tape-spring hinge.

It was mentioned in Sec. II that the tape-spring hinge has to be pinched before one starts to rotate the ends. This process was simulated by defining two rigid plates connected by a single beam element that undergoes thermal contraction. The plates were restrained to the $Y-Z$ plane to maintain symmetry. The connections between the beam element and the rigid plates were modeled as fixed, using CONN3D2 elements and weld connector sections.

The definition of several contact surfaces is also required, as different parts of the tape-spring hinge come into contact with each other, and the rigid plates come into contact with the tape-spring hinge. The general contact feature was assigned to the entire model by specifying contact inclusions, all exterior. With this option, Abaqus/Explicit automatically defines potential contact surfaces around the whole hinge surface. However, the beam element connecting the two plates used for pinching was allowed to go through the hinge by using the contact exclusions parameter.

\section{Abaqus/Explicit Simulation Techniques}

Folding and deployment simulations of ultrathin structures involve significant geometric changes that are associated with instabilities, dynamic snaps, and extensive contact/sliding between different parts of the structure. In previous studies $[\underline{8}, \underline{12}]$, issues of numerical stability and convergence associated with singularity in the stiffness matrix were the main limit on the range of structural configurations that could be studied and the amount of folding that could be imposed on a given structure. To remove these limitations, the present study has adopted an alternative approach that avoids the stiffness matrix and, instead, advances the kinematic state of each degree of freedom by direct integration of its equations of motion. This solution is obtained with the Abaqus/Explicit finite element package [11].

The behavior that is of interest in the present study is quasi static; hence, inertia effects have to be minimized throughout the solution. In principle, this can be achieved by applying any loads sufficiently slowly and smoothly that the kinetic energy in the structure remains negligibly small, but in reality, there are many details that have to be fine tuned to actually achieve this aim. Our first attempts at doing this, based on the extensive guidance provided in the Abaqus manual, were presented in [14] but, in fact, those preliminary results were of limited accuracy when compared with experimental measurements and even showed significant levels of residual strain energy in the structure at the end of a full folding and deployment cycle. Here, we present a much refined implementation of this approach.

We use three independent ways to control our analysis: integration time increment, loading rate, and numerical damping. Next, we explain their effects and what limitations have to be met when one attempts to optimize the related simulation parameters in folding/ deployment studies.

First, the integration time increment should be as large as possible to reduce the number of increments to complete the simulation. However, explicit time integration is stable only if the Courant condition is satisfied $[\underline{17}, \underline{18}]$ : essentially, the time increment cannot be larger than the time for a wave to travel between adjacent nodes in the finite element mesh. Abaqus/Explicit includes damping effects and estimates the stable time increment limit at each time increment from the approximate relationship [17]

$$
\Delta t=\alpha\left(\sqrt{1+\xi^{2}}-\xi\right) \frac{l_{\min }}{c_{d}}
$$

where the wave speed is

$$
c_{d} \approx \sqrt{\frac{E}{\rho}}
$$

Second, any loads should be applied as smoothly as possible, and the loading rate should be as high as possible to minimize the number of integration increments required to complete the analysis, provided that the response of the structure remains quasi static. The smoothness of the load application is achieved with the Abaqus/ Explicit command: *amplitude, definition $=$ smooth step. This prescribes a fifth-order polynomial time variation with first and second time derivatives equal to zero at the beginning and end of the time interval. Regarding the overall loading rate, the key question is how short the simulation time can be made without exciting a significant dynamic response. The first mode that would get excited is the fundamental natural mode of the whole structure and, although its frequency and mode shape will change during folding/ deployment, it is useful to obtain an approximate estimate at the beginning of the simulation. This can easily be done by an eigenvalue analysis of the structure in its initial configuration.

Third, numerical damping is introduced in the model to dissipate energy buildup at high frequencies to avoid the sudden collapse of elements due to large outbalance forces that may develop at a few nodes and to generally keep down the amount of kinetic energy in the structure. The amount of numerical damping should be as small as possible to avoid affecting the results of the simulation. This is particularly critical for ultrathin structures for which the low bending stiffness could easily be swamped by damping effects and to avoid decreasing the stable time increment (note that, in Eq. (9), $\Delta t$ decreases when $\xi$ is increased).

Abaqus/Explicit allows inclusion of damping in several different ways, of which two (bulk viscosity and viscous pressure) are used; these two particular types of damping are explained next.

The first type of damping, bulk viscosity introduces an in-plane strain-rate-dependent pressure

$$
p_{b}=\xi \rho c_{d} l \dot{\epsilon}_{v}
$$

and an in-plane curvature-rate-dependent moment

$$
m=\xi \frac{h^{2}}{12} \rho c_{d} l \dot{\kappa}
$$

both distributed over all shell elements.

The second type of damping, viscous pressure, introduces a velocity-dependent normal pressure over all shell elements:

$$
p=-c_{v} v \cdot n
$$

This normal pressure is very effective in quickly damping out dynamic effects and thus reaches quasi-static equilibrium in a minimal number of increments. However, it is important to use an appropriate value of $c_{v}$; if it is too high, the response of the structure will be overdamped, and hence the simulation results may be incorrect. Note that, unlike bulk viscosity, introducing viscous pressure does not change the integration time increment.

There is an alternative technique, mass scaling, that is often used to speed up explicit analyses. This technique consists of artificially increasing the density of the material to $\beta \rho$ in order to increase the time increment from Eq. (9) to $\sqrt{\beta} \Delta t$. This technique would be useful in simulations involving rate-dependent materials, where the load-rate speedup technique adopted in the present study could not be used, but it offers no advantages in the present case.

The key test for the robustness of a particular analysis is to consider the history of the various energy terms and, in particular, the energy balance history. The energy balance $E_{b}$ is defined as the difference between the energy stored in the structure and/or dissipated during 


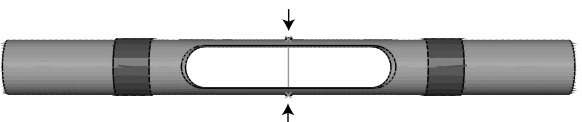

a) Undeformed configuration

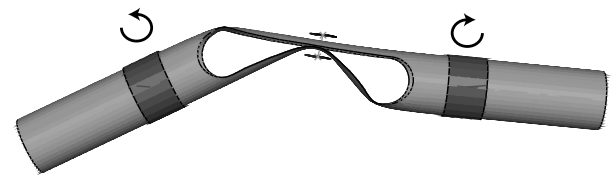

c) Release of contact between hinge and rigid plates

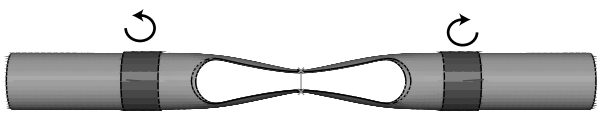

b) Pinching

Fig. 8 Stages of folding simulation.

the loading process, $E_{i}+E_{v}+E_{k}$, and the work of all external forces $E_{w}$. In symbols,

$$
E_{b}=E_{i}+E_{v}+E_{k}-E_{w}
$$

where the internal energy $E_{i}$ is equal to the sum of strain energy and artificial energy (due to hourglassing), $E_{v}$ is the viscous dissipation, and $E_{k}$ is the kinetic energy. In the present simulations, the artificial energy was kept negligibly small by using fully integrated elements and by avoiding any localized actions on the shells.

There are two main checks on the energies. First, at any particular time, the kinetic energy has to be a small fraction (less than 1\%) of the internal energy for the results at that time to be considered a valid quasi-static solution. In the case of multistable structures, if the kinetic energy has reached high levels during an earlier part of the simulation, the possibility that the structure may have reached an alternative equilibrium configuration should be considered. Second, the energy balance should remain equal to the amount of external energy (e.g., thermal) introduced in the system.

In particular, Belytschko et al. [17] discussed cases where an arrested instability in a structure, due to geometric and/or material softening, may have resulted in the integrator losing stability, despite the use of Eq. (9) to compute the time increment. These authors have reported that all such instabilities can be detected by checking the energy balance, as discussed previously.

\section{Simulation of Tape-Spring Hinge}

The fundamental natural period of vibration of the tape-spring hinge in the deployed configuration was computed by doing a frequency analysis in Abaqus/Standard. The estimated period was $5 \mathrm{~ms}$; hence, the definition of the overall loading rate was initially set such that folding/deployment would occur over a time period of 10 times the fundamental period, or $0.05 \mathrm{~s}$. The entire simulation was run with the double precision solver, and the energy components were recorded at every millisecond. The ratio between kinetic energy and internal energy was then monitored, and the loading rate was decreased until $E_{k} / E_{i}<1 \%$.

An example of a successful simulation is presented next to provide a basis for discussion and as a starting point for various sensitivity studies.

The initial, unstressed configuration of the tape-spring hinge is shown in Fig. 8a. At the beginning of the folding simulation, the thermally controlled beam element connecting the two rigid plates was shortened by decreasing its temperature, which simulated the pinching of the tape-spring hinge and led to the configuration in Fig. 8b. The next part of the folding simulation consisted of imposing a rotation of $\theta_{X}^{C}=170 \mathrm{deg}$ over $0.8 \mathrm{~s}$ (this simulation time was obtained by gradually increasing the initial value of $0.05 \mathrm{~s}$, estimated previously). After $0.25 \mathrm{~s}$, corresponding to $\theta_{X}^{C} \approx 16 \mathrm{deg}$ (see Fig. 8c), contact between the rigid plates and the tape-spring hinge was disabled in order to avoid any spurious constraints on the folded shape. The rotation was then continued, and the final outcome is shown in Fig. 8d. Viscous pressure loading, discussed in Sec. V, was used to maintain the energy ratio below $1 \%$.

Figure $\underline{9}$ shows the variation of the energy terms during this simulation. Note that, during the pinching phase, there is an input of thermal energy that is not accounted for in Eq. (14), and this shows as an increase in energy balance. For the rest of the simulation, the energy balance remained approximately constant. Throughout the simulation, the kinetic energy remained much smaller than the internal energy, which indicates that the intended quasi-static behavior of the tape-spring hinge has been achieved.

\section{Setting the Simulation Parameters}

This section presents various sensitivity studies to determine a set of simulation parameters that provide accurate results and maximal speedup of the simulation. These studies were mainly focused on the deployment part of the simulation, because this is the behavior that is of greatest interest for actual applications. However, it was important to first obtain an accurate representation of the folded configuration; hence, two different folding simulations were carried out.

The first folding simulation used no bulk viscosity $(\xi=0)$ but only viscous pressure on both the outer and inner surfaces of the tapespring hinge during the rotational phase; the expression for the pressure is given by Eq. (13) with $c_{v}=2 \times 10^{-4} \rho c_{d}$. The time increment factor in Eq. (9) was set to $\alpha=0.8$. The results have been already presented in Sec. VI, in Fig. 9. Note that the viscous dissipation is zero because the energy absorbed by the viscous pressure is included in the external work. The energy balance remained constant during the rotation phase, which indicates that the simulation was free of instabilities; hence, the results are accurate. The kinetic energy was negligibly small at the end of the simulation,

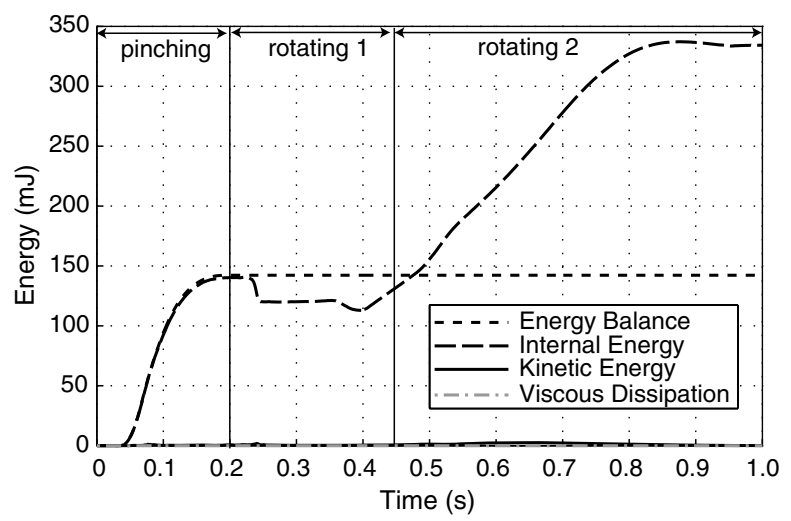

Fig. 9 Energy histories for folding simulation with $\alpha=0.8, \xi=0$, and $c_{v}=2 \times 10^{-4} \rho c_{d}$; the maximum folding angle is $\theta_{X}^{C}=170 \mathrm{deg}$. 


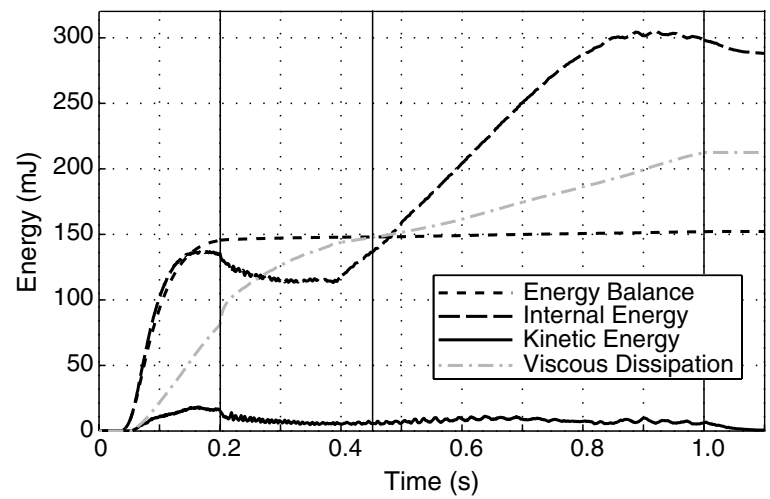

Fig. 10 Energy histories for folding simulation with $\alpha=0.8, \xi=0.10$, and $c_{v}=0$ up to $1.0 \mathrm{~s}$, and then $c_{v}=\times 10^{-2} \rho c_{d}$.

indicating that the folded configuration was in quasi-static equilibrium.

The second folding simulation used bulk viscosity $(\xi=0.10)$ to provide numerical damping. The energy histories are presented in Fig. 10; note the high level of viscous dissipation, representing $70 \%$ of the internal energy at the end of folding phase. Between 1.0 and $1.1 \mathrm{~s}$, viscous pressure was applied (with $c_{v}=2 \times 10^{-2} \rho c_{d}$ ) to decrease the kinetic energy. Reducing the time increment by setting $\alpha$ to less than 1.0 made little difference to these results.

The final configurations predicted by these two folding simulations are approximately equal, but the first simulation was chosen as a starting point for the deployment studies because it has lower kinetic energy and a smoother moment-rotation relationship.

A folded configuration with $\theta_{X}^{C}=160 \mathrm{deg}$ (to avoid the effects of the contact between the ends of the tape-spring hinge), and $\alpha=0.5$, $\xi=0$, and $c_{v}=2 \times 10^{-4} \rho c_{d}$ was chosen as the starting point for several deployment simulations, with different values of the three control parameters.

The sensitivity to $\alpha$ was examined by setting $\alpha=(0.25,0.80)$ with $\xi=0$ and $c_{v}=0$. Figure 11 shows that, in this case, decreasing $\Delta t$ decreases the change in energy balance.

The sensitivity to $\xi$ was examined by setting $\xi=(0,0.01,0.10)$. Figure 11 shows that increasing $\xi$ leads to a decrease of the change in energy balance. Although increasing $\xi$ leads to a small reduction in $\Delta t$, and hence to an increase in the number of simulation increments, it is much more effective in decreasing the energy balance than lowering $\alpha$.

Despite this encouraging result, one should be cautious before relying on bulk viscosity to reduce the energy balance. The problem is that increasing $\xi$ may prevent full deployment. Figure 12 shows the history of the viscous dissipation energy for the three cases that have been considered. It is interesting to note that the viscous dissipation during deployment is 13 and $17 \%$ of the internal energy in the fully folded configuration, respectively, for $\xi=0.01$ and $\xi=0.10$.

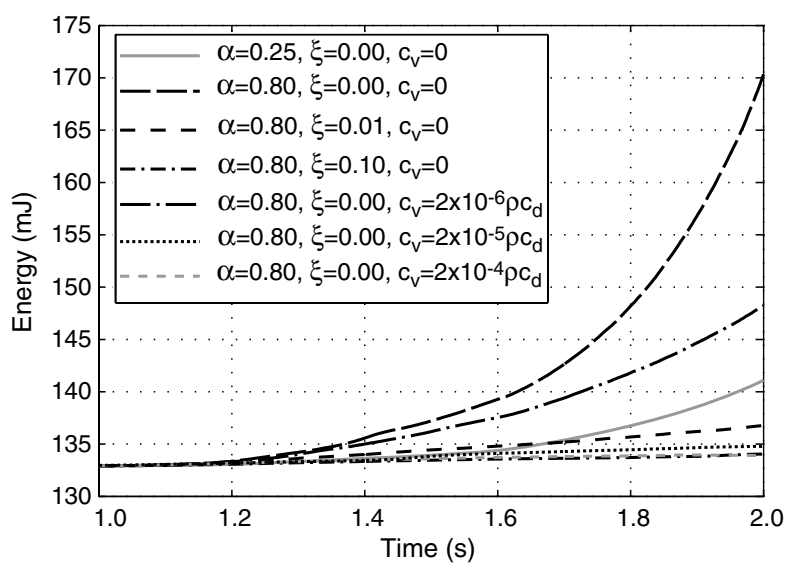

Fig. 11 Energy balance histories for different values of $\alpha, \xi$, and $c_{v}$.
A feature common to the previous simulations, in which bulk viscosity was introduced without viscous pressure, is that there was significant vibration leading to a noisy moment-rotation deployment profile. This issue was addressed by examining the sensitivity to $c_{v}$. Four different values of $c_{v}$ were considered, from 0 to $2 \times 10^{-4} \rho c_{d}$, while maintaining $\alpha=0.8$ and $\xi=0$, and the results are shown in Figs. 11 and 13. Figure 11 shows that the higher values of $c_{v}$ are effective in decreasing the change in energy balance. Figure 13 shows that the particular value $c_{v}=2 \times 10^{-5} \rho c_{d}$ suppresses most tape-spring hinge vibration from $\theta_{X}^{C}=160 \mathrm{deg}$ to about $50 \mathrm{deg}$. However, increasing $c_{v}$ by another order of magnitude radically changes the behavior of the structure.

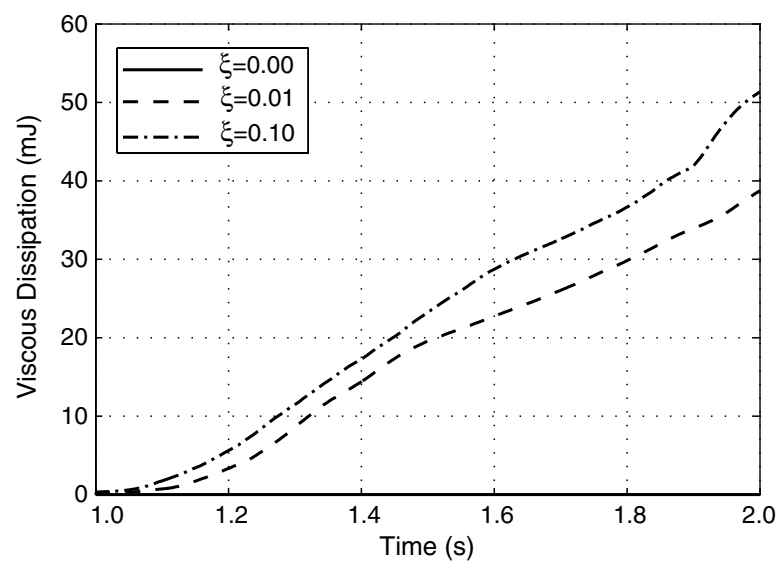

Fig. 12 Variation of viscous dissipation for $\alpha=0.80$ and $c_{v}=0$.

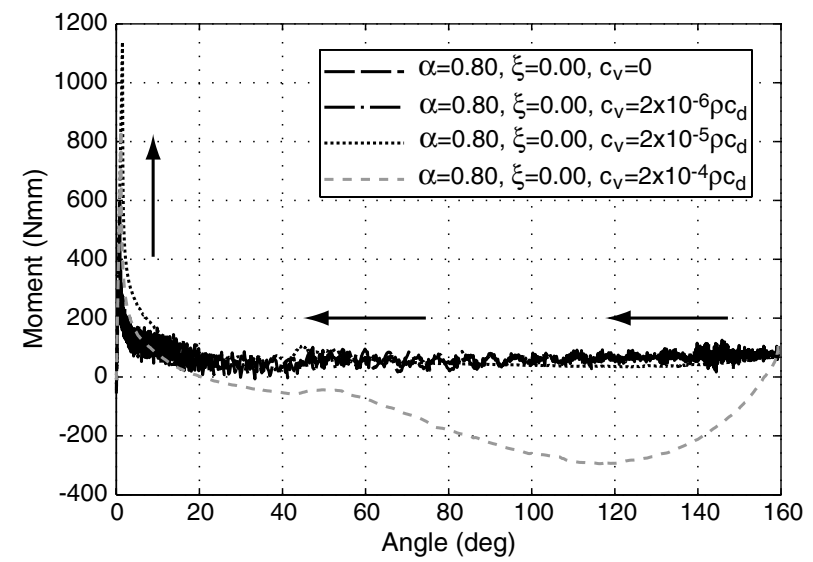

Fig. 13 Sensitivity of moment-rotation response to $c_{v}$ for $\alpha=0.8$ and $\xi=0$. The line style matches Fig. 11 .

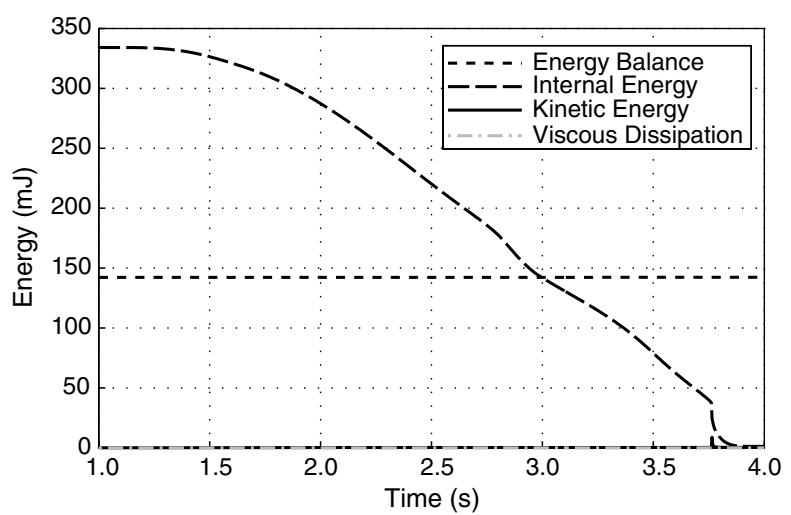

Fig. 14 Energy histories for 3 s deployment with $\alpha=0.8, \xi=0$, and $c_{v}=2 \times 10^{-5} \rho c_{d}$. 

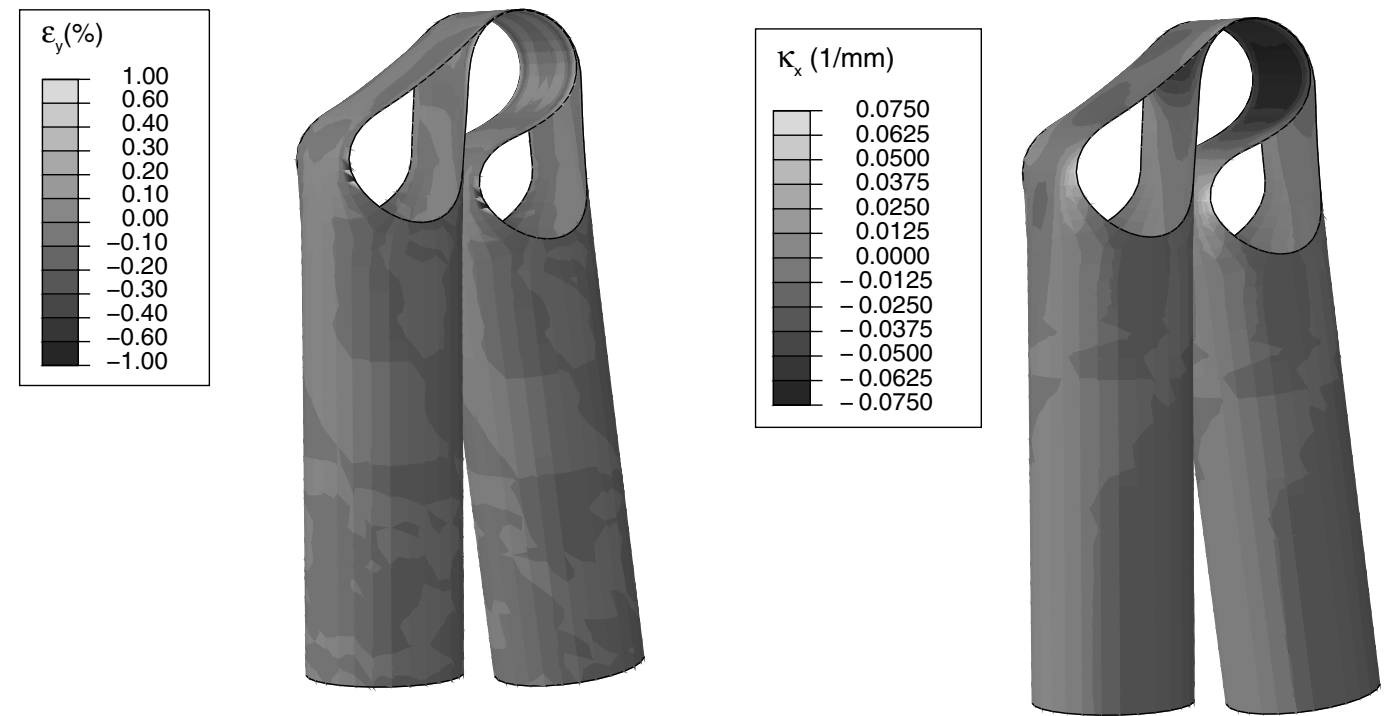

Fig. 15 Midsurface strain and curvature distribution in configuration with $\theta_{X}^{C}=170 \mathrm{deg}$.

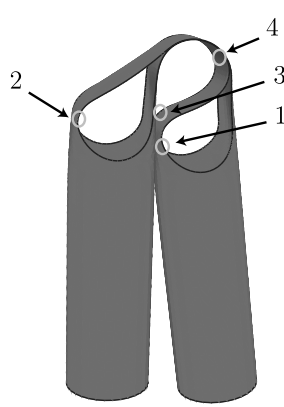

a) Critical regions

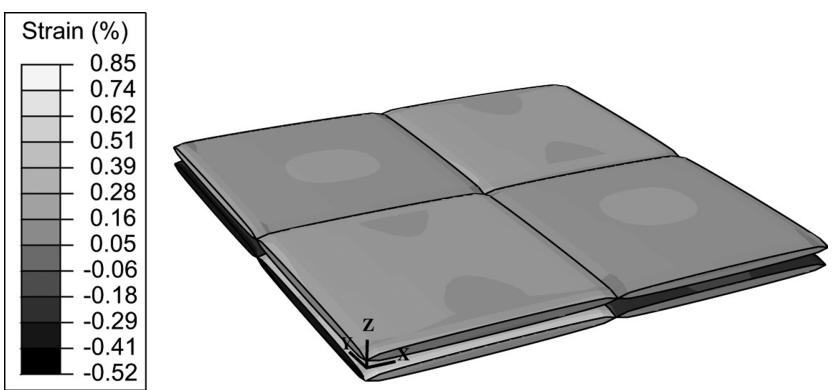

b) Distribution of fiber strain at location 1

Fig. 16 Most highly deformed regions of configuration with $\theta_{X}^{C}=$ $170 \mathrm{deg}$ and contour plot of fiber strains in unit cell at location 1.

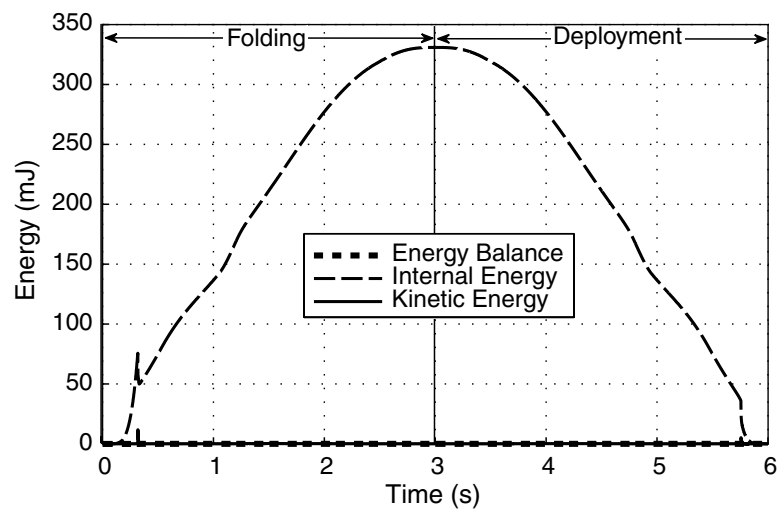

Fig. 17 Energy histories for simulation of folding to $\theta_{X}^{C}=170 \mathrm{deg}$ without pinching and deployment.
In conclusion, the viscous pressure coefficient $c_{v}$ is the most effective tool for keeping the change in energy balance small, but it is important to examine the effects of $c_{v}$ on the structural response in order to avoid overdamping. Based on the study presented in this section, it was decided that the parameters $\alpha=0.8, \xi=0$, and $c_{v}=2 \times 10^{-5} \rho c_{d}$ are best for deployment simulations of our particular hinge.

Once optimal deployment simulation parameters had been obtained, the overall simulation time was increased from 1 to $3 \mathrm{~s}$, and Fig. 14 shows the energy history for this final simulation. Note that this particular simulation started from a folded configuration with $\theta_{X}^{C}=170 \mathrm{deg}$. These results are of very good quality, as the energy balance remains constant throughout the simulation and show a sudden reduction in internal energy at $3.77 \mathrm{~s}$, accompanied by a small spike in kinetic energy. These features correspond to the dynamic snapback of the hinge into its fully deployed configuration. This jump releases practically all the internal energy in the hinge.

\section{Strains in Folded Configuration}

The simulation model provides the midsurface strains and curvatures in the longitudinal and circumferential directions everywhere in the structure and for all configurations. These results can be analyzed to check that a given design of the tape-spring hinge would not be damaged by folding. For example, we will consider the deformation predicted by the first folding simulation presented in Sec. VII but extended to a folding angle $\theta_{X}^{C}=170 \mathrm{deg}$. The midsurface strains and curvature components in the $x$ and $y$ directions (Fig. 5) were obtained from the transformations

$$
\begin{gathered}
\varepsilon_{x}=\sin ^{2} \phi \varepsilon_{x^{\prime}}+\cos ^{2} \phi \varepsilon_{y^{\prime}}-\cos \phi \sin \phi \gamma_{x^{\prime} y^{\prime}} \\
\varepsilon_{y}=\cos ^{2} \phi \varepsilon_{x^{\prime}}+\sin ^{2} \phi \varepsilon_{y^{\prime}}+\cos \phi \sin \phi \gamma_{x^{\prime} y^{\prime}} \\
\gamma_{x y}=+\cos \phi \sin \phi \varepsilon_{x^{\prime}}-\cos \phi \sin \phi \varepsilon_{y^{\prime}}+\left(\cos ^{2} \phi-\sin ^{2} \phi\right) \gamma_{x^{\prime} y^{\prime}}
\end{gathered}
$$

$$
\kappa_{x}=\sin ^{2} \phi \kappa_{x^{\prime}}+\cos ^{2} \phi \kappa_{y^{\prime}}-\cos \phi \sin \phi \kappa_{x^{\prime} y^{\prime}}
$$

Table 3 Maximum half-tow-width strain averages in fibers and matrix at four locations, shown in Fig. 16a

\begin{tabular}{lcccc}
\hline \hline Location & 1 & 2 & 3 & 4 \\
\hline Fibers, \% & 0.80 & 0.86 & 0.88 & 0.64 \\
Matrix, \% & 1.19 & 0.76 & 1.39 & 0.92 \\
\hline \hline
\end{tabular}



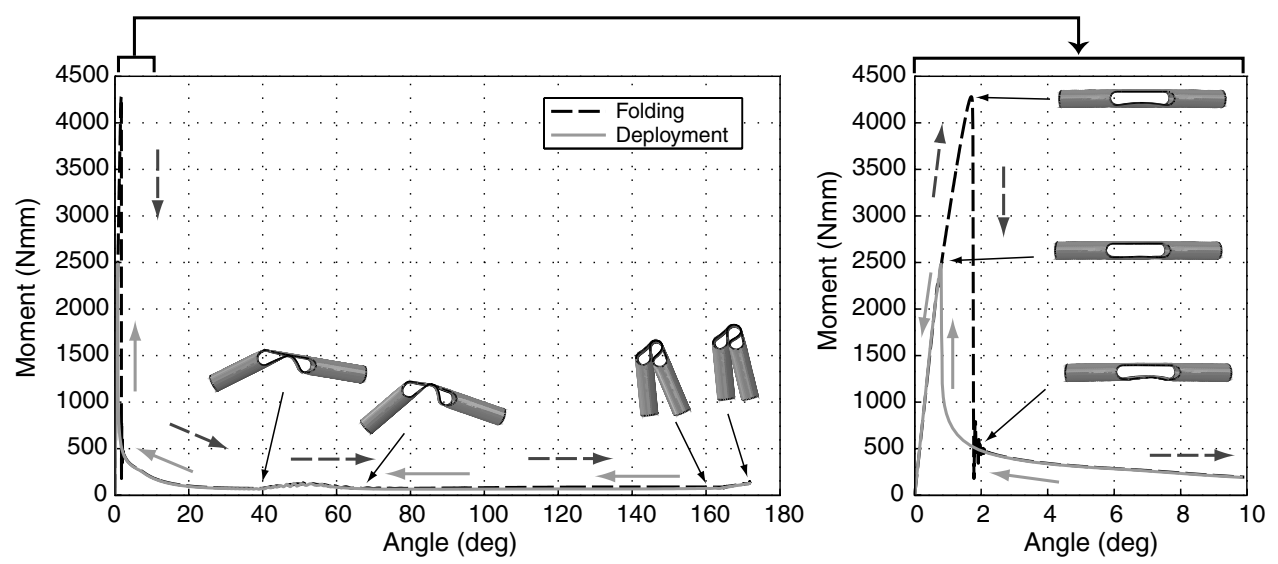

Fig. 18 Moment-rotation profile for folding and deployment simulation (without pinching) up to $\theta_{X}^{C}=170 \mathrm{deg}$.

$$
\begin{gathered}
\kappa_{y}=\cos ^{2} \phi \kappa_{x^{\prime}}+\sin ^{2} \phi \kappa_{y^{\prime}}+\cos \phi \sin \phi \kappa_{x^{\prime} y^{\prime}} \\
\kappa_{x y}=+\cos \phi \sin \phi \kappa_{x^{\prime}}-\cos \phi \sin \phi \kappa_{y^{\prime}}+\left(\cos ^{2} \phi-\sin ^{2} \phi\right) \kappa_{x^{\prime} y^{\prime}}
\end{gathered}
$$

where $\phi=45 \mathrm{deg}$.

Figure 15 shows the midplane strain distribution in the $y$ direction and the curvature in the $x$ direction. Potential failure regions for the fully folded configuration were identified by searching for the largest midplane strains and curvatures (in absolute value): they are shown in Fig. 16a. Six midplane strains and curvatures values were extracted at each location.

These values were substituted into Eqs. (3), and the resulting relative displacements and rotations between the boundary nodes of the periodic unit cell, considered in Sec. III, were used as inputs for four different Abaqus/Standard analyses: one for each critical region identified in Fig. 16a. The results from the first of these analyses are displayed in Fig. $16 \mathrm{~b}$, which shows the distribution of fiber strain corresponding to location 1 .

Following [19], the maximum fiber strain was obtained by computing the average fiber strain in a moving window with a width equal to half a tow width and then selecting the maximum average value. Similarly, the maximum resin strain was obtained by computing 1) the maximum half-tow average normal strain in the direction perpendicular to the fibers in the same moving window and 2) the maximum average principal strain in the resin filling the space between the two plies, and then choosing the maximum of the two.

To establish if the tape-spring hinge is able to withstand this deformation, the maximum normal strain in the fiber direction was compared with the failure strain of T300 fibers (1.5\% ), and the maximum resin strain was compared with the failure strain of HexPly 913 epoxy resin in pure tension. This was calculated to be $1.93 \%$, by dividing the tensile strength $* *$ by the tensile modulus and assuming linear-elastic behavior up to failure. The maximum strains are presented in Table 3, where it can be seen that the hinge has margins of 41 and $28 \%$ against fiber and matrix failure, respectively. Note that the maximum strains occur at the transition between straight and circular slot edges.

\section{Moment-Rotation Relationship}

A complete simulation of the process of folding and deploying the tape-spring hinge was carried out, but this time without initiating the folding process by pinching. The total simulation time was $6 \mathrm{~s}: 3 \mathrm{~s}$ to fold and $3 \mathrm{~s}$ to deploy. The simulation parameters were $\alpha=0.8$, $\xi=0$, and $c_{v}=2 \times 10^{-5} \rho c_{d}$.

\footnotetext{
IData available at http://www.toraycfa.com/pdfs/T300DataSheet.pdf [retrieved 12 November 2010].

**Data available at http://www.hexcel.com/NR/rdonlyres/D2E16C531991-4DD5-8EA2-277ED3323147/0/HexPly_913_eu.pdf [retrieved 12 November 2010].
}

The energy histories have been plotted in Fig. 17. The energy balance remained approximately constant throughout the simulation; hence, the results are accurate. Note that the internal energy profile is mostly symmetric but shows a spike during folding at $0.32 \mathrm{~s}$ that is not matched by a corresponding spike during deployment. In fact, during the initial stages of folding, the strain energy increases at a considerably faster rate than it decreases during the corresponding stages of deployment. An important result is that the internal energy becomes approximately zero at the end of the deployment simulation, indicating that the unstressed, fully deployed configuration has been achieved. Note the kinetic energy spike at $0.32 \mathrm{~s}$ and the smaller spike at $5.76 \mathrm{~s}$; they correspond to the hinge snapping during folding and snapping back, respectively. The viscous dissipation was, of course, zero throughout the entire simulation.

Next, turning to the moment-rotation profile (shown in Fig. 18), the most striking features are that the moment rises to a high peak of $4278 \mathrm{Nmm}$, snaps to about $67 \mathrm{Nmm}$, and remains almost constant from an angle of approximately $20 \mathrm{deg}$ all the way to the fully folded configuration. Note that there is a slight hump between 40 and $60 \mathrm{deg}$, associated with the snapback of the elastic fold at the center of the outer tape spring, and an increase beyond $170 \mathrm{deg}$, when a contact pressure develops between the two ends of the hinge. During deployment, the moment-rotation profile follows the folding profile down to $1.8 \mathrm{deg}$, then it continues to rise gradually, with a final snap back to $2482 \mathrm{Nmm}$ at $0.8 \mathrm{deg}$. It then joins the linear part of the folding response as the angle is further decreased.

This difference between the loading and unloading paths is characteristic of structures with an unstable postbuckling equilibrium path $[20,21]$. It was already know that a single tape spring behaves in this way $[12,22]$, but having established that the postbuckling behavior of tape-spring hinges is also unstable is an interesting result with important implications.

Figure 18 includes snapshots of the deformed configuration just before and soon after snapping back. It is interesting to note that the midsurface strains after the snap are almost zero, with a maximum strain of $0.01 \%$ near the transition between the straight and circular parts of the slot.

\section{Comparison of Experiment and Simulations}

Figure 19 shows a comparison between the deformed configurations of the tape-spring hinge, which were observed during deployment in the test rig, with snapshots from the finite element simulation. This qualitative comparison shows very good agreement in both the positions of the regions of localized deformation and the overall geometric configurations of the tape-spring hinge.

Figure 20 shows a comparison between the deployment momentrotation profile, measured experimentally with the simulated response. Unlike the moment-rotation profile in Fig. 18, the particular simulation results shown here were obtained from a model that included two 5-mm-long aluminum rods, to model the torsional stiffness of the holders. The torsional constant of the rod cross section 


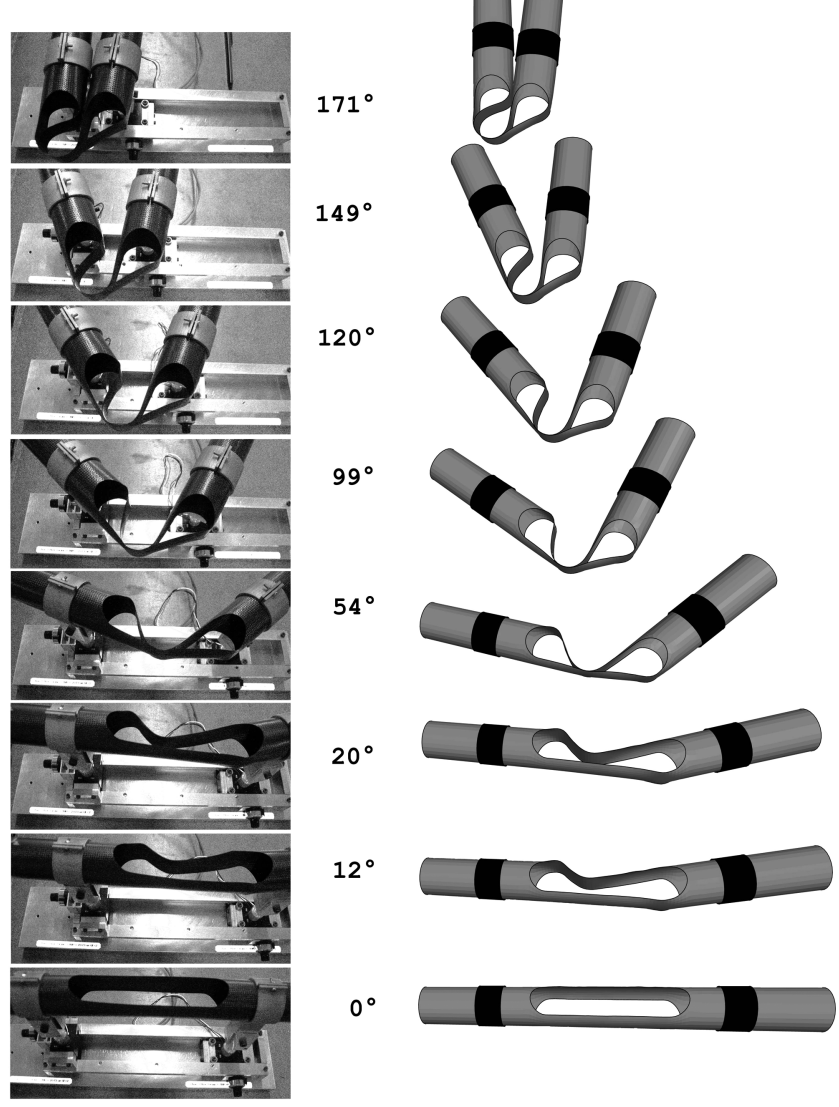

Fig. 19 Comparison of hinge configurations during deployment.

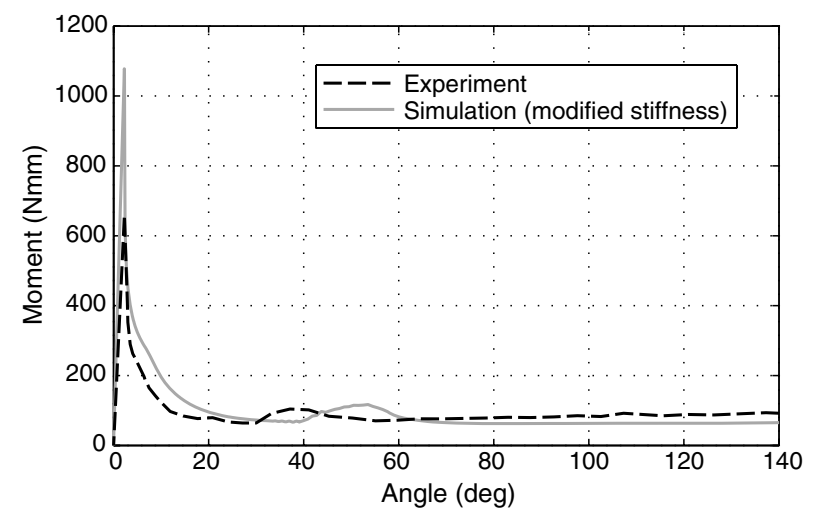

Fig. 20 Comparison of moment-rotation profiles during deployment.

was $12 \mathrm{~mm}^{4}$ to match the angle of snapback observed in the experiment, i.e., $2.3 \mathrm{deg}$.

Overall, the simulation has fully captured the experimentally observed behavior. However, the measured steady-state deployment moment had an average value of $80 \mathrm{Nmm}$, whereas our simulation gave around $67 \mathrm{Nmm}$. Also, the peak in the hump of the experiment was at $\approx 40 \mathrm{deg}$ with a maximum moment of $104 \mathrm{Nmm}$, whereas in the simulation, it was at $\approx 54 \mathrm{deg}$ with a maximum of $117 \mathrm{Nmm}$. Also, the measured peak moment at snapback was $660 \mathrm{Nmm}$, whereas it was significantly higher $(1078 \mathrm{Nmm})$ in the simulation.

\section{Conclusions}

This paper has presented a detailed study of the folding and deployment of a tape-spring hinge made from a two-ply plain-weave laminate of carbon-fiber reinforced plastic. A physical model of a particular version of this hinge was constructed, and its momentrotation profile during quasi-static deployment was measured, starting from the fully folded configuration. The main contribution of this paper is that it has captured the whole process in detail through a finite element simulation, starting from a micromechanical model of the laminate based on the measured geometry and elastic properties of the woven tows. The first stage of this simulation generated the fully folded, strained configuration of the hinge; this was done by pinching the hinge in the middle to reduce the peak moment required to fold the hinge and then rotating the two ends. The second stage in the simulation was to gradually decrease the relative rotation between the ends until it became zero. This process captured both the steady-state-moment part of the deployment, during which a localized fold could be seen in each of the tape springs that made up the hinge, as well as the snap back to the straight configuration.

As an alternative to the simulation of the actual folding process, we have also carried out a simulation of a complete folding and deployment cycle without pinching the hinge. This approach has provided an estimate of the maximum moment that could be carried by the hinge during operation. This alternative simulation has shown that, for our particular hinge design, the deployment moment peak is about half the folding moment peak, a situation typical of deployable structures based on thin shells. The compliance of the testing rig further decreases the deployment moment. From an operational standpoint, the high peak moment for folding indicates that, when the hinge is latched, it is locked in a highly stable configuration; hence, a high pointing accuracy can be expected.

The comparison in Sec. X between the measured and predicted moment-rotation relationships during quasi-static deployment of the tape-spring hinge has shown that, although there is good qualitative agreement, there are three main areas of quantitative discrepancy, as follows. The largest discrepancy was in the magnitude of the snapback moment, overestimated by $63 \%$; in addition, the rotation angle at the hump peak (corresponding to the point of separation of the tape springs) was overestimated by $35 \%$, and the deploymentmoment average was underestimated by $16 \%$. These errors are significantly larger than those obtained when testing the validity of the material model (in Sec. III), but it should be noted that the focus of Sec. III was on the small-strain behavior of the material, and timedependent behavior was not investigated.

It is believed that the main reasons for these discrepancies are the viscoelastic behavior of the composite material (particularly associated with the matrix), tow misalignment, and deadband effects in the testing machine combined with the inability to measure instantaneous response. The first topic is not well understood at present, as it has only recently started to be addressed in deployable structures [23]. The other two issues could be addressed by a more precise manufacturing technique and by the use of a testing rig designed to capture peak values during snapback.

\section{Acknowledgments}

H. M. Y. C. Mallikarachchi thanks the Cambridge Commonwealth Trust and the California Institute of Technology for financial support. Financial support from the Northrop Grumman Corporation and the provision of materials from Hexcel, U.K. are gratefully acknowledged by the authors. The authors also thank Ahmad Kueh, Julian Santiago Prowald, and Michael Sutcliffe for helpful discussions.

\section{References}

[1] Warren, P. A., "Foldable Member," U.S. Patent 374,565 B1, 2002.

[2] Anon., "Hughes Graphite Antennas Installed on MSAT-2 Craft," Space News, Nov. 1994.

[3] Seizt, P., "Spar Resolving Spat Over Antenna Work," Space News, 29 Aug.-4 Sept. 1994.

[4] Adams, D. S., and Mobrem, M., "Lenticular Jointed Antenna Deployment Anomaly and Resolution Onboard the Mars Express Spacecraft," Journal of Spacecraft and Rockets, Vol. 46, No. 2, 2009, pp. $403-410$. doi: $10.2514 / 1.36891$

[5] Soykasap, O., Watt, A., and Pellegrino, S., "New deployable reflector 
concept," 45th AIAA/ASME/ASCE/AHS/ASC Structures, Structural Dynamics, and Materials Conference, Palm Springs, CA, AIAA Paper 2004-1574, 19-22 April 2004.

[6] Jorgensen, J., Louis, E., Hinkle, J., and Silver, M., "Dynamics of an Elastically Deployable Solar Array: Ground Test Model Validation," 46th AIAA/ASME/ASCE/AHS/ASC Structures, Structural Dynamics, and Materials Conference, Austin, TX, AIAA Paper 2005-1942, 18 21 April 2005.

[7] Soykasap, O., Pellegrino, S., Howard, P., and Notter, M., "Folding Large Antenna Tape Spring," Journal of Spacecraft and Rockets, Vol. 45, No. 3, 2008, pp. 560-567. doi: $10.2514 / 1.28421$

[8] Yee, J. C. H., and Pellegrino, S., "Composite Tube Hinges," Journal of Aerospace Engineering, Vol. 18, No. 4, 2005, pp. 224-231. doi:10.1061/(ASCE)0893-1321(2005)18:4(224)

[9] Soykasap, O., "Deployment Analysis of a Self-Deployable Composite Boom," Composite Structures, Vol. 89, No. 3, 2009, pp. 374-381. doi:10.1016/j.compstruct.2008.08.012

[10] Mobrem, M., and Adams, D. S., "Deployment Analysis of Lenticular Jointed Antennas Onboard the Mars Express Spacecraft," Journal of Spacecraft and Rockets, Vol. 46, No. 2, 2009, pp. 394-402. doi: $10.2514 / 1.36890$

[11] Abaqus/Explicit, Software, Ver. 6.7, Simulia, Providence, R.I., 2007.

[12] Seffen, K. A., and Pellegrino, S., "Deployment Dynamics of Tape Springs," Proceedings of the Royal Society of London A, Vol. 455, No. 1983, 1999, pp. 1003-1048. doi:10.1098/rspa.1999.0347

[13] Soykasap, O., "Micromechanical Models for Bending Behaviour of Woven Composites," Journal of Spacecraft and Rockets, Vol. 43, No. 5, 2006, pp. 1093-1100. doi: $10.2514 / 1.18010$

[14] Mallikarachchi, H. M. Y. C., and Pellegrino, S., "Simulation of QuasiStatic Folding and Deployment of Ultra- Thin Composite Structures," 49th AIAA/ASME/ASCE/AHS/ASC Structures, Structural Dynamics, and Materials Conference, Schaumburg, IL, AIAA Paper 2008-2053, 07-10 April 2008.
[15] Karkkainen, R. L., and Sankar, B. V., "A Direct Micromechanics Method for Analysis of Failure Initiation of Plain Weave Textile Composites," Composites Science and Technology, Vol. 66, No. 1, 2006, pp. $137-150$. doi:10.1016/j.compscitech.2005.05.018

[16] Kueh, A. B. H., and Pellegrino, S., " $A B D$ Matrix of Single-Ply Triaxial Weave Fabric Composites," 48th AIAA/ASME/ASCE/AHS/ASC Structures, Structural Dynamics, and Materials Conference, Honolulu, HI, AIAA Paper 2007-2161, 23-26 April 2007.

[17] Belytschko, T., Liu, W. K., and Moran, B., "6.2 Explicit Methods," Nonlinear Finite Elements for Continua and Structures, Wiley, New York, 2000, pp. 315-316.

[18] Geradin, M., and Rixen, D., Mechanical Vibrations: Theory and Application to Structural Dynamics, Wiley, New York, 1994.

[19] Yang, Q. D., Rugg, K. L., Cox, B. N., and Marshall, D. B., "Evaluation of Macroscopic and Local Strains in a Three-Dimensional Woven C/ SiC Composite," Journal of the American Ceramic Society, Vol. 88, No. 3, 2005, pp. 719-725. doi:10.1111/j.1551-2916.2005.00156.x

[20] Brush, D. O., and Almroth, B. O., Buckling of Bars, Plates and Shells, McGraw-Hill, New York, 1975.

[21] van der Heijden, A. M. A., W.T. Koiter's Elastic Stability of Solids and Structures, Cambridge Univ. Press, New York, 2009.

[22] Warren, P. A., Dobson, B. J., Hinkle, J. D., and Silver, M. "Experimental Characterization of Lightweight Strain Energy Deployment Hinges," 46th AIAA/ASME/ASCE/AHS/ASC Structures, Structural Dynamics and Materials Conference, Austin, TX, AIAA Paper 2005-1809, 18-21 April 2005.

[23] Kwok, K., and Pellegrino, S., "Shape Recovery of Viscoelastic Deployable Structures," 51st AIAA/ASME/ASCE/AHS/ASC Structures, Structural Dynamics and Materials Conference, Orlando, FL, AIAA Paper 2010-2606, 12-15 April 2010.
L. Peterson Associate Editor 